Aleksandar Milašinović

Zdravko Milovanović

Darko Knežević

Indir Mujanić

DOI: $10.21278 /$ TOF.40207

ISSN 1333-1124

eISSN 1849-1391

\title{
DETERMINATION OF DIFFERENTIAL EQUATIONS OF MOTION AND PARAMETERS OF AN ELASTIC INTERNAL COMBUSTION ENGINE CRANKSHAFT
}

\begin{abstract}
Summary
The goal of this paper is to develop mathematically less complex differential equations of motion of an elastic crankshaft and their solutions with acceptable accuracy in relation to the actual system. The instantaneous angular speed of the free end of the crankshaft consists of the nonuniform rigid body motion mode and elastic deformation mode. In general, the crankshaft, as well as other parts of the engine, could be considered as a structural component with distributed mass and elasticity. This will lead to a system with an infinite number of degrees of freedom, and require solving partial differential equations. Another approach is to discretize the continuous system into a finite set of rigid bodies interconnected with springs and dampers, which is the method chosen here. The lumped mass model of the crankshaft and the corresponding differential equations of motion for each mass simulate the actual dynamics of the crankshaft fairly accurately.
\end{abstract}

Key words: $\quad$ crankshaft dynamics, nonuniform angular speed, torsional deformation, lumped mass

\section{Introduction}

The idea of using a lumped mass model for the crankshaft dynamics is conceptually straightforward [4], [5], [7] and [8]. Machines containing reciprocating elements have some characteristic dynamic problems. In many cases, their solution is difficult and designers cannot ensure working conditions as smooth as those typical of machines containing only rotating elements. IC engines are based on the crank mechanism in the form of a crankshaft with several connecting rods and reciprocating elements. IC engines cannot, in general, be exactly balanced. The variability of the equivalent moment of inertia $J_{e q}\left(\omega_{0} t\right)$ generates inertia torques $\frac{1}{2} \frac{d J_{e q}\left(\omega_{0} t\right)}{d\left(\omega_{0} t\right)} \omega_{0}{ }^{2}$ in reciprocating machines. Torsional torque is excited on the crankshaft by inertia variation, whose effect may be considerable. The geometric configuration of reciprocating elements is complex, and crankshafts not only do not possess 
axial symmetry but often do not have symmetry planes. The external forces acting on the crankshaft are variable in time, with periodic law. Also, the forces exerted by gas-pressure on the pistons and other forces typical of reciprocating machines are periodic. The external torque and the crankshaft speed are periodic functions of the crank angle and may be approximated by a Fourier series. Under steady state operating conditions, the variation of the reciprocating inertia torque may be considered identical for all cylinders and shifted in the crank-angle domain according to the firing order of the engine. The gas-pressure torque may differ from cylinder to cylinder, but under steady state operating conditions, the gaspressure torque of a given cylinder undergoes deviation from cycle to cycle.

The dynamic response of the crankshaft to the external torque may be determined by summing up the responses to each harmonic component of the torque. The possibilities of resonance between these forcing functions and the natural frequencies of the system are numerous. The running of an IC engine is characterized - even when operating under steady state conditions - by a cycle's dispersion which has as a consequence the fact that two consecutive cycles of the same cylinder are not identical. It is known that in order to establish a mean reference cycle, the average of more than 100 cycles - for a spark ignition engine - and of about 50 cycles - for a diesel engine - is necessary. These cyclic fluctuations invalidate the hypothesis of truly steady state operating conditions $[4,9]$.

The dynamic response of the crankshaft results from the superposition of the responses to each harmonic component of the resultant engine torque. While the higher harmonic components of the gas pressure torque may excite torsional vibrations of the crankshaft at different engine speeds, the frequencies of the lower harmonic components are much lower than the first natural frequency of the crankshaft at all operating speeds of the engine. The possibilities of resonance between higher harmonic components of the gas pressure forcing functions and the natural frequencies of the system are numerous.

\section{Crankshaft dynamics}

There are many ways to determine the equations of motion of a crankshaft. In this paper, an approach based on the principles of analytical dynamics, using the Lagrange equations of the second kind, is presented.

Besides the specific mathematical apparatus to model motion, analytical mechanics uses a specific physical basis. Unlike the Newtonian vectorial approach, which relies on momentum and force as vector quantities, analytical mechanics is based on scalar properties of motion - energy and work. The main difference between analytical and Newtonian mechanics lies in different ways of modelling the impact of constraints on the motion of a system. A schematics of the lumped mass model of a crankshaft with all regarding parameters could be presented as in Fig.1.

The instantaneous angular speed of the crankshaft is variable due to the variability of the gas-pressure torque and an inertia torque. The motion of the crankshaft is analysed by assuming it is under steady state motion, i.e. constant mean rotational speed.

The generalized coordinates, which define the motion of the lumped mass model of the crankshaft, are crank angles (angular positions) of individual lumped masses $\alpha_{i}(t)$. 


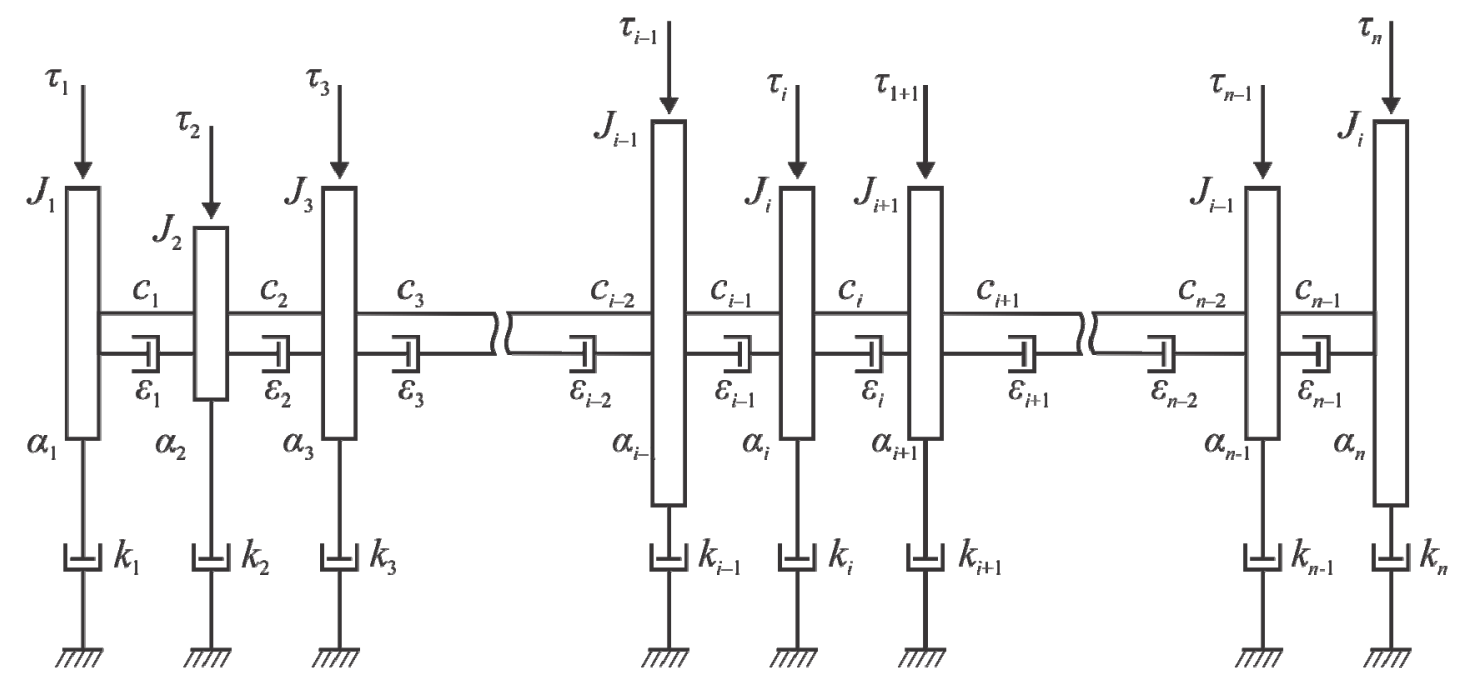

Fig. 1 The torsional dynamic model of the crankshaft

In order to obtain a clearer picture of the crank angle of the $i$-th lumped mass, the crankshaft can be imagined as motionless while being affected by variable driving torques. In this case, the crankshaft has a pure oscillatory motion, i.e. the massless elastic shaft elements are torsionally deformed. As for the real moving system the situation is more complex. The instantaneous angular speed of the crankshaft is variable and there are two possible approaches. The motion of the crankshaft can be observed as two combined movements: the motion of the crankshaft as a rigid body and the torsional deformation. This approach is complex due to the variability of instantaneous angular speed of the crankshaft as a rigid body $[4,16]$. Another approach is to observe the motion of the crankshaft as a motion with mean (constant) angular speed and to add the variable part of motion of every lumped mass. In this case, the nonuniform angular speed of the $i$-th lumped mass $\dot{\varphi}_{i}(t)$ consist of the crankshaft's nonuniform rigid body motion mode and elastic deformation mode. If all lumped masses rotate simultaneously - without torsional deformation, the potential energy (energy stored in the massless elastic shaft elements) will not change, and this is referred to as the model having a rigid body mode. Other modes of motion are called deformational modes or elastic modes.

The differential equations of motion for holonomic systems are the Lagrange equations of the second kind:

$$
\frac{d}{d t}\left(\frac{\partial T}{\partial \dot{\boldsymbol{\alpha}}}\right)-\frac{\partial T}{\partial \boldsymbol{\alpha}}=-\frac{\partial V}{\partial \boldsymbol{\alpha}}-\frac{\partial \Phi}{\partial \dot{\boldsymbol{\alpha}}}+\boldsymbol{\tau}_{l},
$$

where $\frac{d}{d t}\left(\frac{\partial T}{\partial \dot{\boldsymbol{\alpha}}}\right)-\frac{\partial T}{\partial \boldsymbol{\alpha}}$ is the row vector of generalized inertial forces, $\frac{\partial V}{\partial \boldsymbol{\alpha}}$ the row vector of generalized conservative forces, $\frac{\partial \Phi}{\partial \dot{\boldsymbol{\alpha}}}$ the row vector of generalized dissipative forces and $\boldsymbol{\tau}_{l}$ the row vector of generalized non-conservative external forces. The above mentioned vector of generalized external forces refers to a vector comprising generalized forces on each lumped mass, regardless of whether these forces are external forces or constraint forces. For example, if we observe the lumped mass model of the crankshaft, there is no external force acting on the first lumped mass - the flywheel -, but there is a generalized force, which is the result of the constraint force acting between the flywheel and the brake. Frictional forces are not included in the vector of external excitation forces (they are included in the damping matrix through external damping). 
A crankshaft with $n$ lumped masses, rotating at steady state operating conditions, is observed. The angular position of each lumped mass is defined by a column vector of generalized coordinates $\boldsymbol{\alpha}=\left[\alpha_{1}, \alpha_{2}, \ldots, \alpha_{n}\right]^{T}$.

The kinetic energy of the lumped mass model of the crankshaft may be expressed as

$$
T=\frac{\dot{\boldsymbol{\alpha}}^{T} \mathbf{J}(\boldsymbol{\alpha}) \dot{\boldsymbol{\alpha}}}{2}
$$

where $\mathbf{J}(\boldsymbol{\alpha})$ is the moments of inertia matrix of the lumped mass model of the crankshaft.

The potential energy can be expressed as

$$
V=\frac{\boldsymbol{\alpha}^{T} \mathbf{C} \boldsymbol{\alpha}}{2}
$$

where $\mathbf{C}$ is the stiffness matrix of the lumped mass model of the crankshaft.

For the purpose of modelling the motion of an engine crankshaft, typically both absolute and relative damping elements are used. The dissipation function has the following form

$$
\Phi=\frac{\dot{\boldsymbol{\alpha}}^{T} \mathbf{K} \dot{\boldsymbol{\alpha}}}{2}
$$

where $\mathbf{K}$ is the damping matrix.

After substituting particular functional derivatives into the Lagrange equation (1.1) the equation of motion is obtained

$$
\mathbf{J}(\boldsymbol{\alpha}) \ddot{\boldsymbol{\alpha}}+\frac{1}{2} \frac{d \mathbf{j}(\boldsymbol{\alpha})}{d \boldsymbol{\alpha}} \dot{\boldsymbol{\alpha}}^{2}+\mathbf{K} \dot{\boldsymbol{\alpha}}+\mathbf{C} \boldsymbol{\alpha}=\boldsymbol{\tau}_{l},
$$

where is $\mathbf{j}(\boldsymbol{\alpha})=\left[\begin{array}{llll}J_{1}\left(\alpha_{1}\right) & J_{2}\left(\alpha_{2}\right) & \ldots & J_{n}\left(\alpha_{n}\right)\end{array}\right]^{T}$ vector axial moment of inertia, $\frac{d \mathbf{j}(\boldsymbol{\alpha})}{d \boldsymbol{\alpha}}$ diagonal matrix of derivatives axial moments of inertia.

The motion of the lumped mass model of the crankshaft is observed as being composed of the motion of all lumped masses with constant speed together and the relative motion of every lumped mass $\varphi(t)$ in relation to the common crank angle of uniform motion $\boldsymbol{\omega}_{0} t$ of each lumped mass. The following relations, regarding angular position, angular speed and angular acceleration, can be expressed as

$$
\begin{gathered}
\boldsymbol{\alpha}(t)=\boldsymbol{\omega}_{0} t+\boldsymbol{\varphi}(t) \\
\dot{\boldsymbol{\alpha}}(t)=\boldsymbol{\omega}_{0}+\dot{\boldsymbol{\varphi}}(t), \\
\ddot{\boldsymbol{\alpha}}(t)=\ddot{\boldsymbol{\varphi}}(t)
\end{gathered}
$$

where $\boldsymbol{\omega}_{\mathbf{0}}=\left[\omega_{0}, \ldots, \omega_{0}\right]_{1 \times n}^{T}$ is a column vector of mean angular speed, $t$ is time (a scalar quantity) while $\boldsymbol{\varphi}(t)=\left[\varphi_{1}(t), \ldots, \varphi_{n}(t)\right]_{1 \times n}^{T}$ is a column vector of the angle of nonuniform motion of the lumped mass model of the crankshaft. All quantities, which are functions of the vector of the crank angle of uniform motion $\omega_{0} t$, may be approximated by a Taylor series. 
Expanding the functions $\mathbf{j}(\boldsymbol{\alpha}), \frac{d \mathbf{j}(\boldsymbol{\alpha})}{d \boldsymbol{\alpha}}$ and $\boldsymbol{\tau}_{l}(\boldsymbol{\alpha})$ around $\boldsymbol{\omega}_{0} t$ gives

$$
\begin{gathered}
\mathbf{j}(\boldsymbol{\alpha})=\mathbf{j}\left(\boldsymbol{\omega}_{0}+\boldsymbol{\varphi}(t)\right) \approx \mathbf{j}\left(\boldsymbol{\omega}_{0} t\right)+\frac{d \mathbf{j}\left(\boldsymbol{\omega}_{0} t\right)}{d\left(\boldsymbol{\omega}_{0} t\right)} \boldsymbol{\varphi}(t) \\
\frac{d \mathbf{j}(\boldsymbol{\alpha})}{d \boldsymbol{\alpha}} \approx \frac{d \mathbf{j}\left(\boldsymbol{\omega}_{0} t\right)}{d\left(\boldsymbol{\omega}_{0} t\right)}+\frac{d^{2} \mathbf{j}\left(\boldsymbol{\omega}_{0} t\right)}{d\left(\boldsymbol{\omega}_{0} t\right)^{2}} \boldsymbol{\varphi}, \\
\boldsymbol{\tau}_{l}(\boldsymbol{\alpha}) \approx \boldsymbol{\tau}_{l}\left(\omega_{0} t\right) \\
\dot{\boldsymbol{\alpha}}^{2} \approx \boldsymbol{\omega}_{0}^{2}+2 \boldsymbol{\omega}_{0} \dot{\boldsymbol{\varphi}}
\end{gathered}
$$

This choice of terms of the Taylor series is a result of knowing the process, more precisely, knowing the order of magnitude of the mean crank angle of uniform motion $\omega_{0} t$ and the row vector of the angle of nonuniform motion $\varphi$ of every lumped mass of the equivalent dynamic model of the crankshaft $\left(\left|\varphi_{i}\right| \ll\left|\omega_{o} t\right|, i=1,2, \ldots, n\right)$.

The first simplification is to replace a $\mathbf{J}\left(\boldsymbol{\omega}_{0} t+\boldsymbol{\varphi}\right) \ddot{\boldsymbol{\varphi}}$ in the term $\mathbf{J}\left(\boldsymbol{\omega}_{0} t\right) \ddot{\boldsymbol{\varphi}}$ for the actual value that is a function of $\boldsymbol{\omega}_{0} t+\boldsymbol{\varphi}$. Equations 1.7 and 1.5, while omitting $\frac{d^{2} \mathbf{j}\left(\boldsymbol{\omega}_{0} t\right)}{d\left(\boldsymbol{\omega}_{0} t\right)^{2}} \boldsymbol{\omega}_{0} \dot{\boldsymbol{\varphi} \varphi} \boldsymbol{\varphi}$ as a lower-order term, together yield a system of differential equations in the form of a matrix

$$
\mathbf{J}\left(\boldsymbol{\omega}_{0} t\right) \ddot{\boldsymbol{\varphi}}+\mathbf{K} \dot{\boldsymbol{\varphi}}+\frac{d \mathbf{j}\left(\boldsymbol{\omega}_{0} t\right)}{d\left(\boldsymbol{\omega}_{0} t\right)} \boldsymbol{\omega}_{0} \dot{\boldsymbol{\varphi}}+\frac{1}{2} \frac{d^{2} \mathbf{j}\left(\boldsymbol{\omega}_{0} t\right)}{d\left(\boldsymbol{\omega}_{0} t\right)^{2}} \boldsymbol{\omega}_{0}^{2} \boldsymbol{\varphi}+\mathbf{C} \boldsymbol{\varphi}=\boldsymbol{\tau}_{l}\left(\boldsymbol{\omega}_{0} t\right)-\frac{1}{2} \frac{d \mathbf{j}\left(\boldsymbol{\omega}_{0} t\right)}{d\left(\boldsymbol{\omega}_{0} t\right)} \boldsymbol{\omega}_{0}^{2}-\mathbf{K} \boldsymbol{\omega}_{0} .
$$

After analyzing equation 1.8 , it can be noticed that the torque is composed of the torque referring to external torque acting on all lumped masses $\boldsymbol{\tau}_{l}\left(\boldsymbol{\omega}_{0} t\right)$ and the torque due to the motion of the slider-crank mechanism $\frac{1}{2} \frac{d \mathbf{j}\left(\boldsymbol{\omega}_{0} t\right)}{d\left(\boldsymbol{\omega}_{0} t\right)} \boldsymbol{\omega}_{0}^{2}$. The term $\mathbf{K} \boldsymbol{\omega}_{\mathbf{0}}$ is the constant friction torque. The moment of inertia and its first and second derivative are periodic functions of the crank angle of uniform motion $\boldsymbol{\omega}_{0} t$ and, for the given system of differential equations, they are variable coefficients, i.e. the system is nonlinear. The term $\frac{1}{2} \frac{d^{2} \mathbf{j}\left(\boldsymbol{\omega}_{0} t\right)}{d\left(\boldsymbol{\omega}_{0} t\right)^{2}} \boldsymbol{\omega}_{0}^{2} \boldsymbol{\varphi}$ takes into consideration the torque variation, caused by the masses of the slider-crank mechanism, with the angle of nonuniform motion $\boldsymbol{\varphi}(t)$ of the lumped mass model of the crankshaft. Equation 1.8 takes the variability of the equivalent moment of inertia into consideration as well as the variability of torque with the angle of nonuniform motion $\varphi(t)$. For the purpose of practical analysis of the motion of the crankshaft, equation 1.8 is linearized and becomes

$$
\mathbf{J}_{\text {mean }} \ddot{\boldsymbol{\varphi}}+\mathbf{K} \dot{\boldsymbol{\varphi}}+\mathbf{C} \boldsymbol{\varphi}=\boldsymbol{\tau}_{l}\left(\boldsymbol{\omega}_{0} t\right)-\frac{1}{2} \frac{d \mathbf{j}\left(\boldsymbol{\omega}_{0} t\right)}{d\left(\boldsymbol{\omega}_{0} t\right)} \boldsymbol{\omega}_{0}^{2}-\mathbf{K} \boldsymbol{\omega}_{\mathbf{0}}
$$

where $\mathbf{J}_{\text {mean }}$ is the moment of inertia matrix of the system with mean components which are not depending on the crank angle of uniform motion $\boldsymbol{\omega}_{0} t$. 
The moment of inertia of lumped masses which are not part of the slider-crank mechanism is constant and can be easily determined on the basis of a solid model (e.g. flywheel, pulley). The situation is more complex when it comes to the parts of the crankshaft where elements that drive auxiliary devices are connected (e.g. camshaft, injection pump...). In this case, the moment of inertia has to be reduced to the crankshaft axis. The moment of inertia of lumped mass is in this case also constant. When it comes to lumped masses regarding the slider-crank mechanism, the situation is more complex. The equivalent moment of inertia is variable and a function of the crank angle of uniform motion. Detailed analysis can be found in $[4,5]$.

Fig. 2 shows a slider-crank mechanism. The whole system can be modelled from the viewpoint of its kinetic energy by the moment of inertia of a single crankshaft crank, rotating at the angular speed $\omega_{0}$.

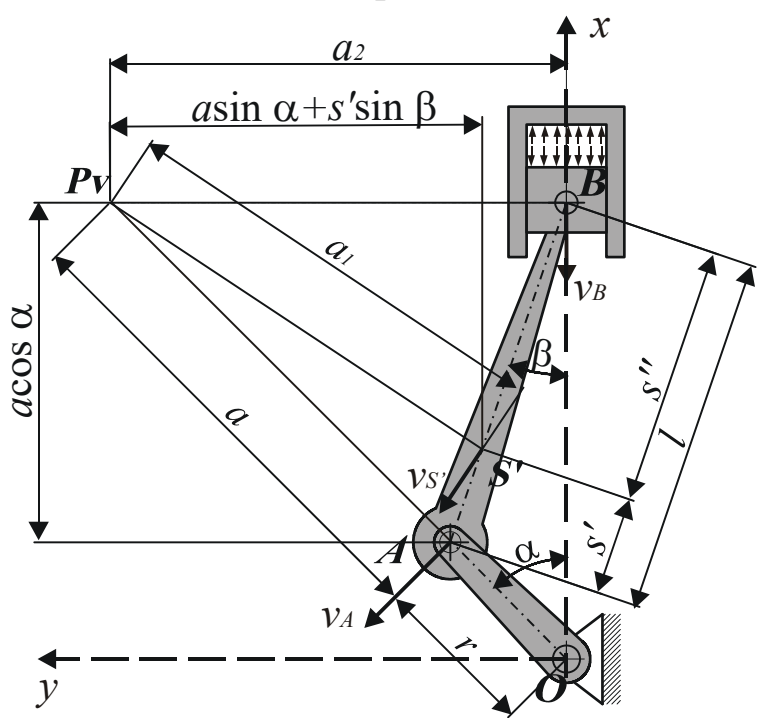

Fig. 2 Slider-crank mechanism

The equivalent moment of inertia of a slider-crank mechanism is a periodic function with a period of $2 \pi$ and can be expressed in a Fourier series [16]

$$
J_{\text {eq }}\left(\omega_{0} t\right)=J_{\text {mean }}+\left(m_{\text {rec }}+m_{B}\right) r^{2} \sum_{k=1}^{n} a_{k} \cos k\left(\omega_{0} t\right)
$$

where $J_{\text {mean }}=J_{c r}+m_{A} r^{2}+a_{0}\left(m_{\text {rec }}+m_{B}\right) r^{2}$ is the mean equivalent moment of inertia of the slider-crank mechanism, and the Fourier coefficients $a_{k}$ are determined by the equation

$$
\left.\begin{array}{c}
a_{0}=\frac{1}{2}+\frac{1}{8} \lambda^{2}+\frac{1}{16} \lambda^{4}+\frac{5}{128} \lambda^{6}+\frac{7}{256} \lambda^{8}+\ldots \\
a_{1}=\frac{1}{2} \lambda+\frac{1}{8} \lambda^{3}+\frac{15}{256} \lambda^{5}+\frac{35}{1024} \lambda^{7}+\ldots \\
a_{2}=-\frac{1}{2}-\frac{1}{32} \lambda^{4}-\frac{1}{32} \lambda^{6}-\frac{7}{256} \lambda^{8}-\ldots \\
a_{3}=-\frac{1}{2} \lambda-\frac{3}{16} \lambda^{3}-\frac{27}{256} \lambda^{5}-\frac{35}{512} \lambda^{7}-\ldots \\
a_{4}=-\frac{1}{8} \lambda^{2}-\frac{1}{16} \lambda^{4}-\frac{1}{32} \lambda^{6}-\frac{1}{64} \lambda^{8}-\ldots \\
a_{5}=\frac{1}{16} \lambda^{3}+\frac{15}{256} \lambda^{5}+\frac{25}{512} \lambda^{7}+\ldots \\
a_{6}=\frac{1}{32} \lambda^{4}+\frac{1}{32} \lambda^{6}+\frac{13}{512} \lambda^{8}+\ldots \\
a_{7}=\frac{-3}{256} \lambda^{5}-\frac{35}{2048} \lambda^{7}-\ldots \\
a_{8}=-\frac{1}{128} \lambda^{6}-\frac{3}{256} \lambda^{8}-\ldots
\end{array}\right\}
$$


The expression for the equivalent moment of inertia of the $j$-th slider-crank mechanism has the form

$$
J_{e q_{j}}\left(\omega_{0} t\right)=J_{\text {mean }_{j}}+\sum_{k=1}^{n} J_{A_{j k}} e^{\mathrm{i} k \omega_{0} t}+\sum_{k=1}^{n} J_{B_{j k}} e^{-\mathrm{i} k \omega_{0} t}
$$

where

$$
\begin{aligned}
& J_{A_{j k}}=\frac{a_{k}\left(m_{r e c}+m_{B}\right) r^{2} \cos k \delta_{j}-i a_{k}\left(m_{r e c}+m_{B}\right) r^{2} \sin k \delta_{j}}{2}, \\
& J_{B_{j k}}=\frac{a_{k}\left(m_{k g}+m_{B}\right) r^{2} \sin k \delta_{j}+i a_{k}\left(m_{k g}+m_{B}\right) r^{2} \cos k \delta_{j}}{2}, \\
& i=\sqrt{-1}, \delta_{j} \text { - phase on the } j \text {-th crank. }
\end{aligned}
$$

The equation of motion (1.8) in the matrix form is

$$
\begin{gathered}
\mathbf{J}_{\text {mean }} \ddot{\boldsymbol{\varphi}}+\sum_{k=1}^{n}\left[\mathbf{J}_{\mathbf{A}_{k}} e^{i k \omega_{0} t}+\mathbf{J}_{\mathbf{B}_{k}} e^{-i k \omega_{0} t}\right] \ddot{\boldsymbol{\varphi}}+ \\
+\mathbf{K} \dot{\boldsymbol{\varphi}}+\omega_{0} i \sum_{k=1}^{n} k\left[\mathbf{J}_{\mathbf{A}_{k}} e^{i k \omega_{0} t}-\mathbf{J}_{\mathbf{B}_{k}} e^{-i k \omega_{0} t}\right] \dot{\boldsymbol{\varphi}}- \\
-\frac{1}{2} \omega_{0}^{2} \sum_{k=1}^{n} k^{2}\left[\mathbf{J}_{\mathbf{A}_{k}} e^{i k \omega_{0} t}+\mathbf{J}_{\mathbf{B}_{k}} e^{-i k \omega_{0} t}\right] \boldsymbol{\varphi}+\mathbf{C} \boldsymbol{\varphi}= \\
=\boldsymbol{\tau}_{l}\left(\boldsymbol{\omega}_{0} t\right)-\frac{1}{2} i \omega_{0}^{2} \sum_{k=1}^{n} k\left[\mathbf{J}_{\mathbf{A}_{k}} \mathbf{i} e^{i k \omega_{0} t}-\mathbf{J}_{\mathbf{B}_{k}} \mathbf{i} e^{-i k \omega_{0} t}\right]-\mathbf{K \boldsymbol { \omega } _ { \mathbf { 0 } }}
\end{gathered}
$$

The mean equivalent moment of inertia is in the form of a diagonal matrix while the stiffness matrix and the damping matrix are tridiagonal matrices. $\mathbf{J}_{\mathbf{A}_{k}}$ and $\mathbf{J}_{\mathbf{B}_{k}}$ are diagonal matrices and comprise the coefficients $J_{A_{j k}}$ and $J_{B_{j k}}$; the column vector $\mathbf{i}$ is a unit vector $\mathbf{i}=[1, \ldots, 1]_{1 \times n}^{T}$. Equation 1.13 is the general form of the crankshaft equation of motion which takes the variability of the equivalent moment of inertia into consideration. By solving the homogeneous part of the system of differential equations with constant coefficients, which describe the motion, natural frequencies are gained. However, in the case of systems with variable coefficients, although the external excitation is equal to zero, the existence of functions of time in the homogeneous part of the equations acts like excitation. This kind of excitation acts from the system parameters and is usually called parametric excitation. By solving the homogeneous part of the system of differential equations with variable coefficients, the interval of stability of motion is determined [16].

It has been shown that neglecting the variability of the moment of inertia leads to very small errors [16, 17]. Therefore, for the purpose of an engineering analysis a modified equation, obtained from equation 1.13, while neglecting the variability of the moment of inertia of the slider-crank mechanism, could be used

$$
\mathbf{J}_{\text {const }} \ddot{\boldsymbol{\varphi}}+\mathbf{K} \dot{\boldsymbol{\varphi}}+\mathbf{C} \boldsymbol{\varphi}=\boldsymbol{\tau}_{l}\left(\boldsymbol{\omega}_{0} t\right)-\frac{1}{2} i \omega_{0}^{2} \sum_{k=1}^{n} k\left[\mathbf{J}_{\mathbf{A}_{k}} \mathbf{i} e^{i k \omega_{0} t}-\mathbf{J}_{\mathbf{B}_{k}} \mathbf{i} e^{-i k \omega_{0} t}\right]-\mathbf{K} \boldsymbol{\omega}_{\mathbf{0}}
$$




\section{Parameters of the lumped mass model of the crankshaft}

In order to be able to solve the equation of motion of a crankshaft (1.14) it is necessary to know the parameters of the mathematical model. The considered parameters of the system are: the moment of inertia matrix $\mathbf{J}$, the damping matrix $\mathbf{K}$, the stiffness matrix $\mathbf{C}$, the vector of external torque $\boldsymbol{\tau}_{l}$ and the vector of the variability of the equivalent moment of inertia of the slider-crank mechanism $\frac{1}{2} \frac{d \mathbf{j}\left(\boldsymbol{\omega}_{0} t\right)}{d\left(\boldsymbol{\omega}_{0} t\right)} \boldsymbol{\omega}_{0}^{2}$ which represents the excitation due to inertial torque of the system. In most cases, the determination of the system parameters cannot be done accurately and directly. The determination of the moment of inertia matrix is an example of when it is possible to quite accurately determine the parameters of the system. Moments of inertia of the bodies constituting the dynamic system of the lumped mass model of the crankshaft can be determined on the basis of created solid models. The accuracy of this method depends on the accuracy of the solid models. The stiffness of cylindrical parts of the crankshaft, which do not comprise a crank, can be accurately determined by applying FEM on the created solid model. Although stiffness of particular parts of the crankshaft cannot be accurately determined, the interval related to the stiffness can be located. The detailed procedure of the indirect determination of the stiffness of the crankshaft crank, based on measuring the angular speed of the free end of the crankshaft, is given in [15]. In this paper, the engine friction is modelled as viscous damping, which is proportional to the angular speed of the corresponding lumped mass, also called absolute damping. The value of the absolute damping was experimentally determined. The absolute damping coefficient $k$ was determined by the measurement of brake power and the constant part of the torque which is gained by a harmonic analysis of the indicated pressure. The braking load can be approximated as constant $[4,9,10,11,18]$. Furthermore, it can be assumed that this torque is acting on the last mass of the equivalent dynamic model.

\section{Result analysis}

A turbocharged low-speed diesel engine with direct fuel injection was experimentally investigated. The main characteristics of the tested engine are presented in Table 1.

Table 1 Characteristics of the tested engine: TAM BF 4 L 515 C

\begin{tabular}{|l|c|c|c|}
\hline Property & Symbol & Value & Unit \\
\hline Piston head area & $A_{k}$ & 0.123 & $\mathrm{~m}^{2}$ \\
\hline Crank radius & $r$ & 0.0725 & $\mathrm{~m}$ \\
\hline Length of the connection rod & $l$ & 0.237 & $\mathrm{~m}$ \\
\hline Firing order & & $1-3-4-2$ & \\
\hline Reciprocating mass & $m_{r e c}$ & 4.6 & $\mathrm{~kg}$ \\
\hline Rotating mass & $m_{r o t}$ & 2.6 & $\mathrm{~kg}$ \\
\hline Cylinder capacity & $V$ & 7000 & $\mathrm{~cm}^{3}$ \\
\hline Bore diameter & $d$ & 125 & $\mathrm{~mm}$ \\
\hline Stroke & $s$ & 145 & $\mathrm{~mm}$ \\
\hline Engine power / rpm & $P / n$ & $150 / 2150$ & $\mathrm{~kW} / \mathrm{min}^{-1}$ \\
\hline Max engine torque $/ \mathrm{rpm}$ & $T / n$ & $787 / 1500$ & $\mathrm{Nm} / \mathrm{min}^{-1}$ \\
\hline
\end{tabular}


Determination of Differential Equations of Motion and Parameters of an Elastic

Internal Combustion Engine Crankshaft
A. Milašinović, Z. Milovanović, D. Knežević, I. Mujanić

Fig. 3.a shows the measured and modelled angular speed of the pulley as well as the angular speed of the flywheel, calculated for $1000 \mathrm{rpm}$. As it can be noticed, the shapes of the measured and calculated angular velocities are quite well matching but there are significant differences in amplitude. This is a direct consequence of the assumption that the torque of the last mass of the equivalent dynamic model is constant. Also, on the curve of angular speed of the pulley oscillations of greater frequency can be noticed. These oscillations result from the torsional deformation of the pulley relative to the flywheel. Since being an equivalent mass of greater moment of inertia in relation to the pulley and the slider-crank mechanism, the flywheel acts as a filter which does not allow oscillations of great frequency to pass. Fig. 3.b shows the angle of nonuniform motion of an equivalent mass corresponding to the pulley and flywheel as well as the torsional (angular) deformations between them. As it can be noticed, the nonuniform rotation of the crankshaft is dominant. Despite operating under maximum engine load, elastic deformations of the crankshaft are negligible for the aforementioned speed regime.

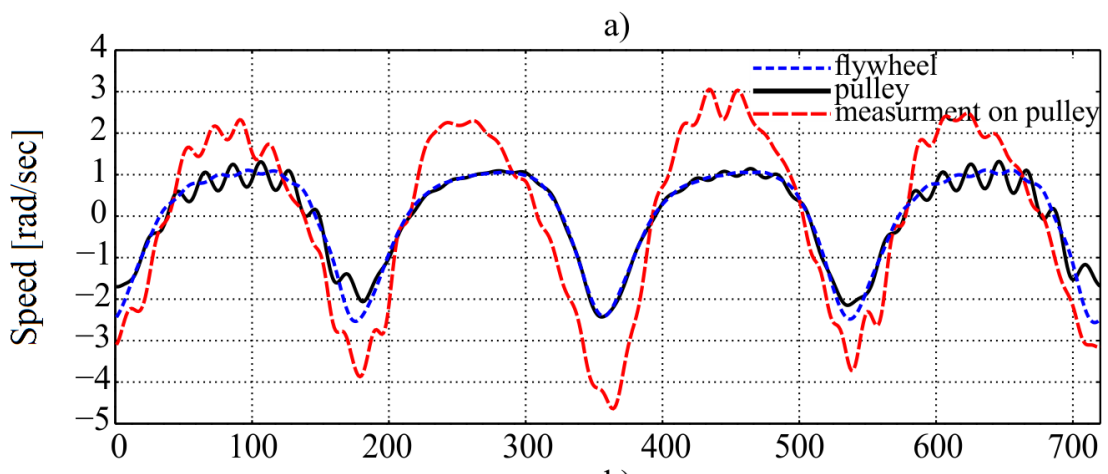

b)

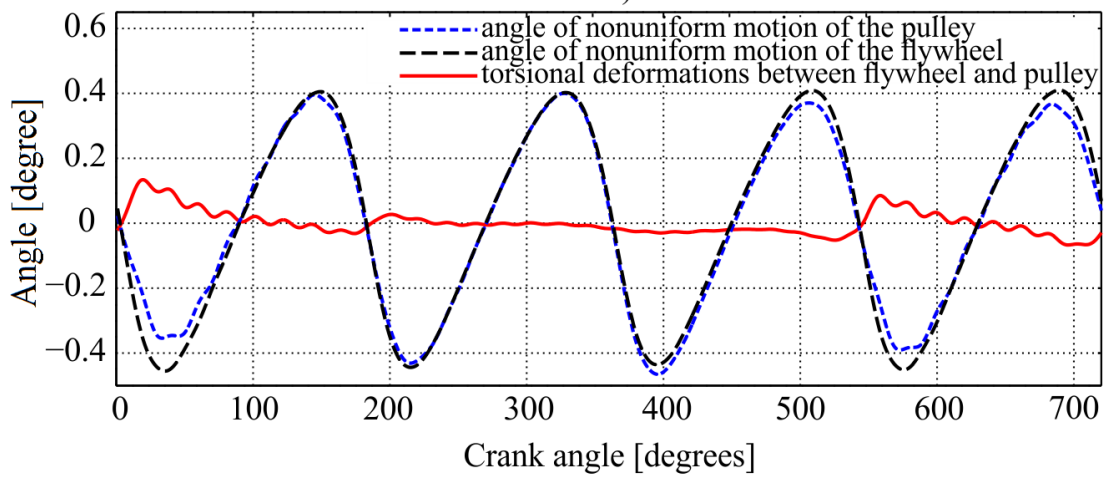

Fig. 3 Calculated angular speed of flywheel and pulley and measured angular speed of pulley (a); angular position of flywheel and pulley and modelled torsional (angular) deformations between them (b) at $1000 \mathrm{rpm}$

Fig. 4 shows amplitudes of the angle of nonuniform motion of the flywheel, the angle of nonuniform motion of the pulley and torsional (angular) deformations at $1000 \mathrm{rpm}$. As it can be noticed, the main harmonics of the $2 \mathrm{nd}$, 4th and 6th order are dominant. They generally cause nonuniform rigid body rotation of the crankshaft. However, from torsional deformation harmonic analysis it can be seen that rigid body mode are also dominant when it comes to the elastic deformation of the crankshaft. Also, the influence of the 16th, 16.5th, 17.5th and 18th harmonic can be noticed. They cause the deformational motion, Fig 4.b.

Fig. 5.a shows the measured and modelled angular speed of the pulley as well as the angular speed of the flywheel, calculated for $1500 \mathrm{rpm}$. To better match the measured and the calculated angular speed it would be necessary to consider the excitation torque at the flywheel. 

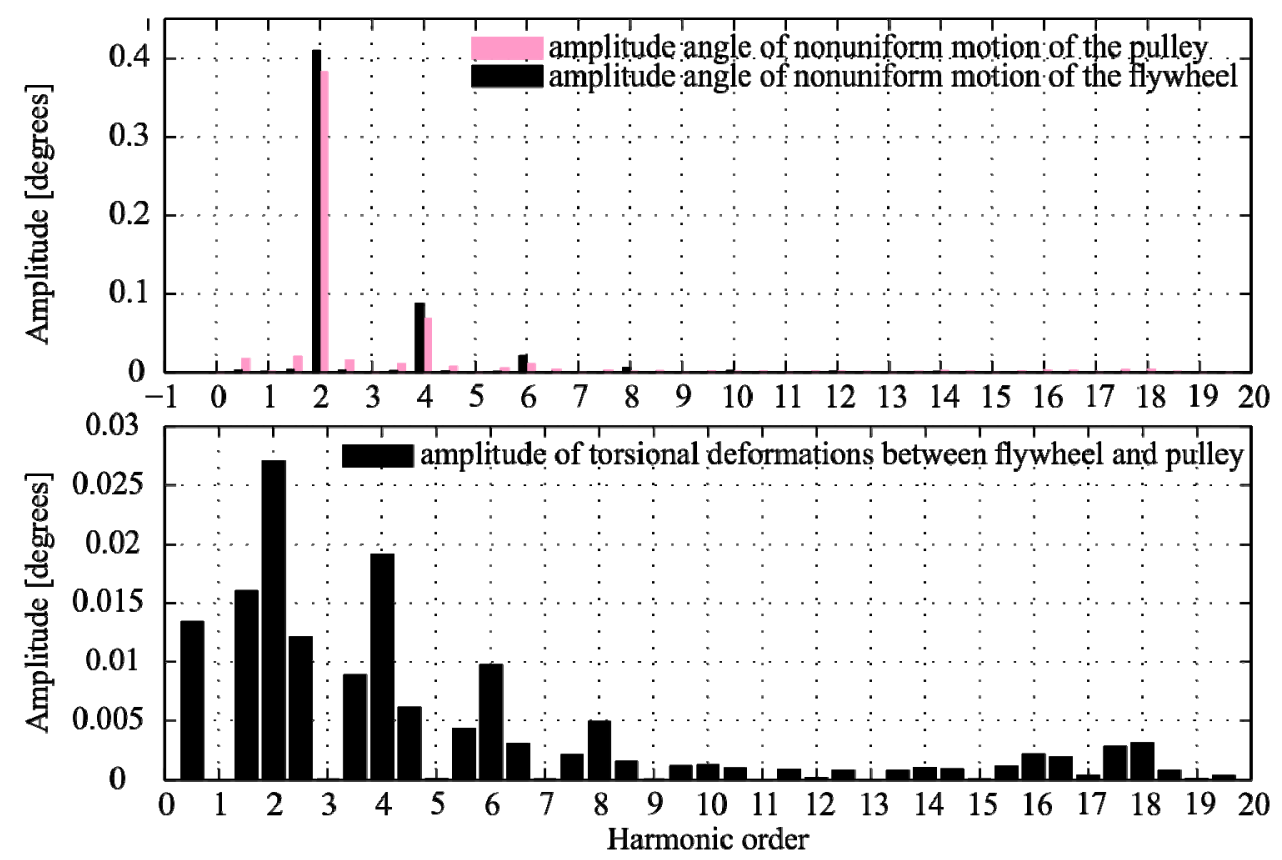

Fig. 4 Calculated amplitudes of particular harmonics of angle of nonuniform motion of flywheel, angle of nonuniform motion of pulley a); and torsional (angular) deformations b) at $1000 \mathrm{rpm}$

It can be noticed that the nonuniform rigid body motion of the crankshaft decreased in comparison to the nonuniform rigid body motion of the crankshaft at $1000 \mathrm{rpm}$ but it is still dominant. The torsional deformation of the pulley relative to the flywheel is greater. The flywheel lowers the amplitude of the rigid body motion of the crankshaft while the curves of angular speed and the nonuniform angle of rotation are smooth in comparison to the curves corresponding to the pulley.
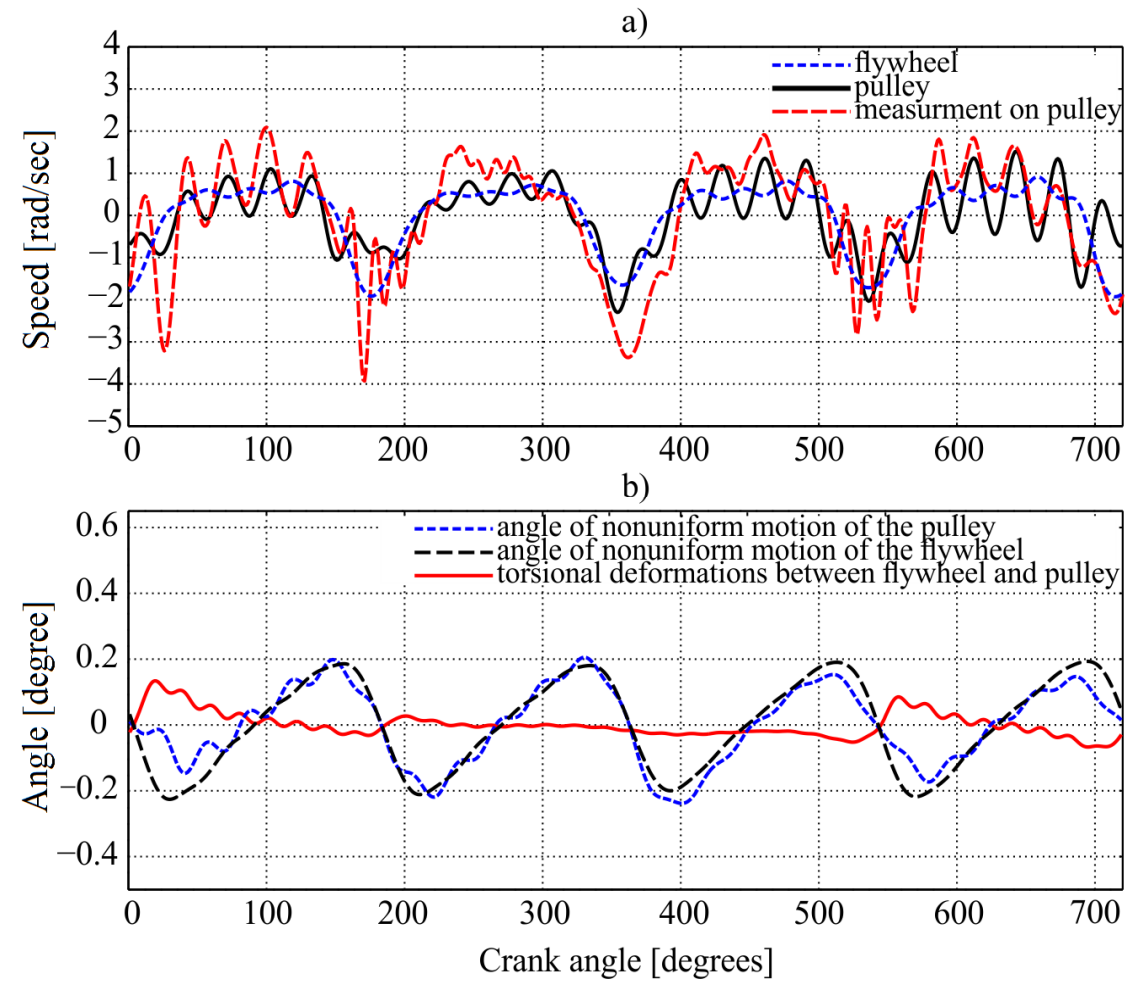

Fig. 5 Calculated angular speed of flywheel and pulley and measured angular speed of pulley (a); angular position of flywheel and pulley and modelled torsional (angular) deformations between them (b) at $1500 \mathrm{rpm}$ 
Fig. 6 shows amplitudes of the angle of nonuniform motion of the flywheel, the angle of nonuniform motion of the pulley and torsional (angular) deformations at $1500 \mathrm{rpm}$. The main harmonics of the $2 \mathrm{nd}$, 4 th and 6 th order contribute to the nonuniform rigid body motion of the crankshaft and cause elastic deformations of the crankshaft. At $1500 \mathrm{rpm}$, the 12th order harmonic contributes significantly to the elastic deformation of the crankshaft.
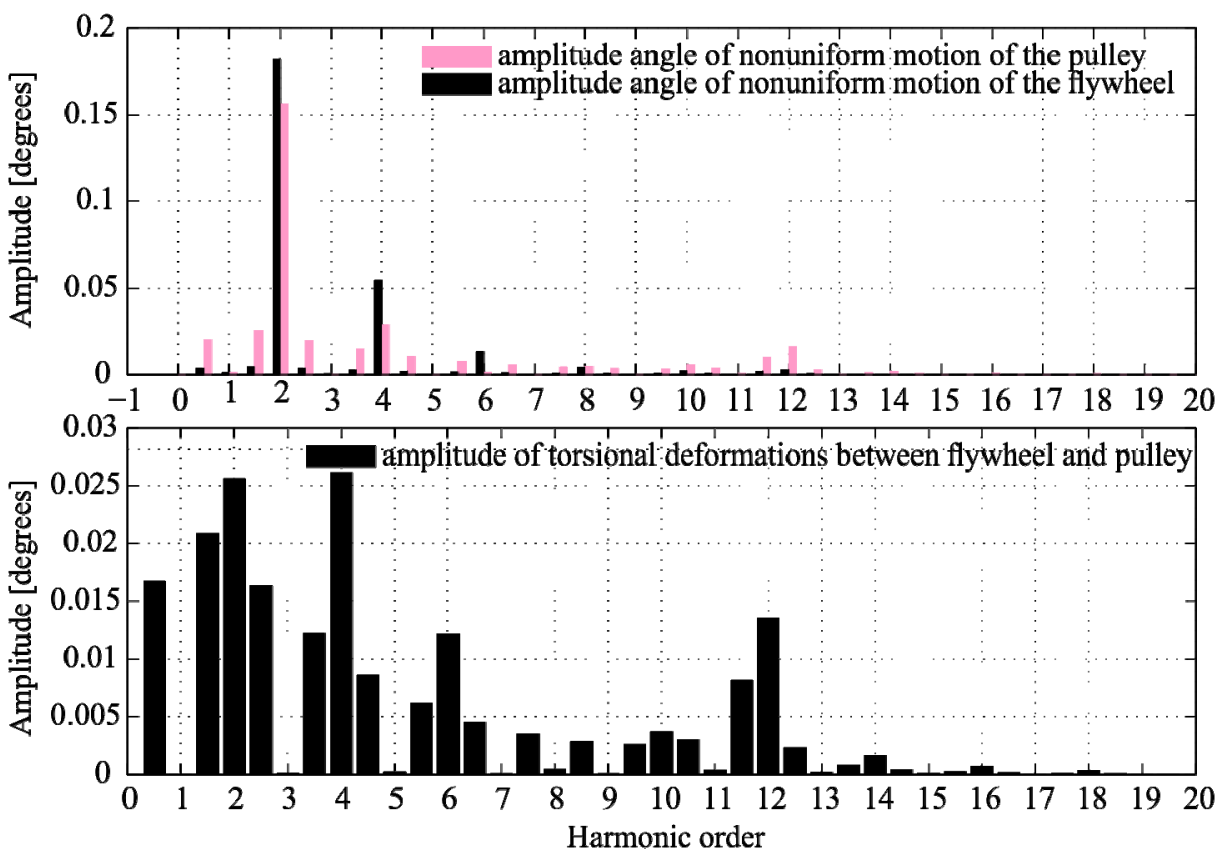

Fig. 6 Calculated amplitudes of particular harmonics of angle of nonuniform motion of flywheel, angle of nonuniform motion of pulley a); and torsional (angular) deformations b); at $1500 \mathrm{rpm}$

\section{Conclusions}

Based on the application of the presented mathematical model of motion of the crankshaft on a concrete example the following may be concluded:

- Taking the variability of the equivalent moment of inertia of the slider-crank mechanism into account leads to nonlinear differential equations of motion of the crankshaft. In contrast to the approach with the constant moment of inertia, when equations can be separately solved for every harmonic, in this case the equations are coupled and have to be solved simultaneously for all harmonics. It has been shown that this leads to not so significant errors - in the case of the concrete engine less than $2 \%$.

- Defining the parameters of the mathematical model represents a crucial step in the mathematical modelling of the crankshaft motion. There are two types of parameters: those which can be determined directly and those which are determined indirectly, based on the analysis of experimental results. Moments of inertia of lumped masses are parameters that can be determined on the basis of solid models. Stiffness and damping of the system are parameters to be estimated on the basis of the analysis of experimental results of instantaneous angular speed of the free end of the crankshaft. Damping should be observed as relative and absolute. Absolute damping should be determined by the measurement of the brake power. Internal damping can be identified during resonant regimes of the running engine.

- The angle of nonuniform motion of the lumped mass comprises the elastic deformation and the nonuniform rigid body motion of the crankshaft. Nonuniform rigid body motion of the crankshaft is dominant at lower speed regimes of the shaft, 
while the elastic deformation is dominant at higher speed regimes. The elastic deformation of a massless elastic shaft element should be defined when the angle of nonuniform motion of the lumped mass on the left-hand side is subtracted from the angle of nonuniform motion of the lumped mass on the right-hand side of the same massless elastic shaft element.

- The correct determination of excitation would require the measurement of indicated pressures in all cylinders and the determination of the excitation torque acting on the last mass of the equivalent dynamic model towards the brake.

\section{Nomenclature}

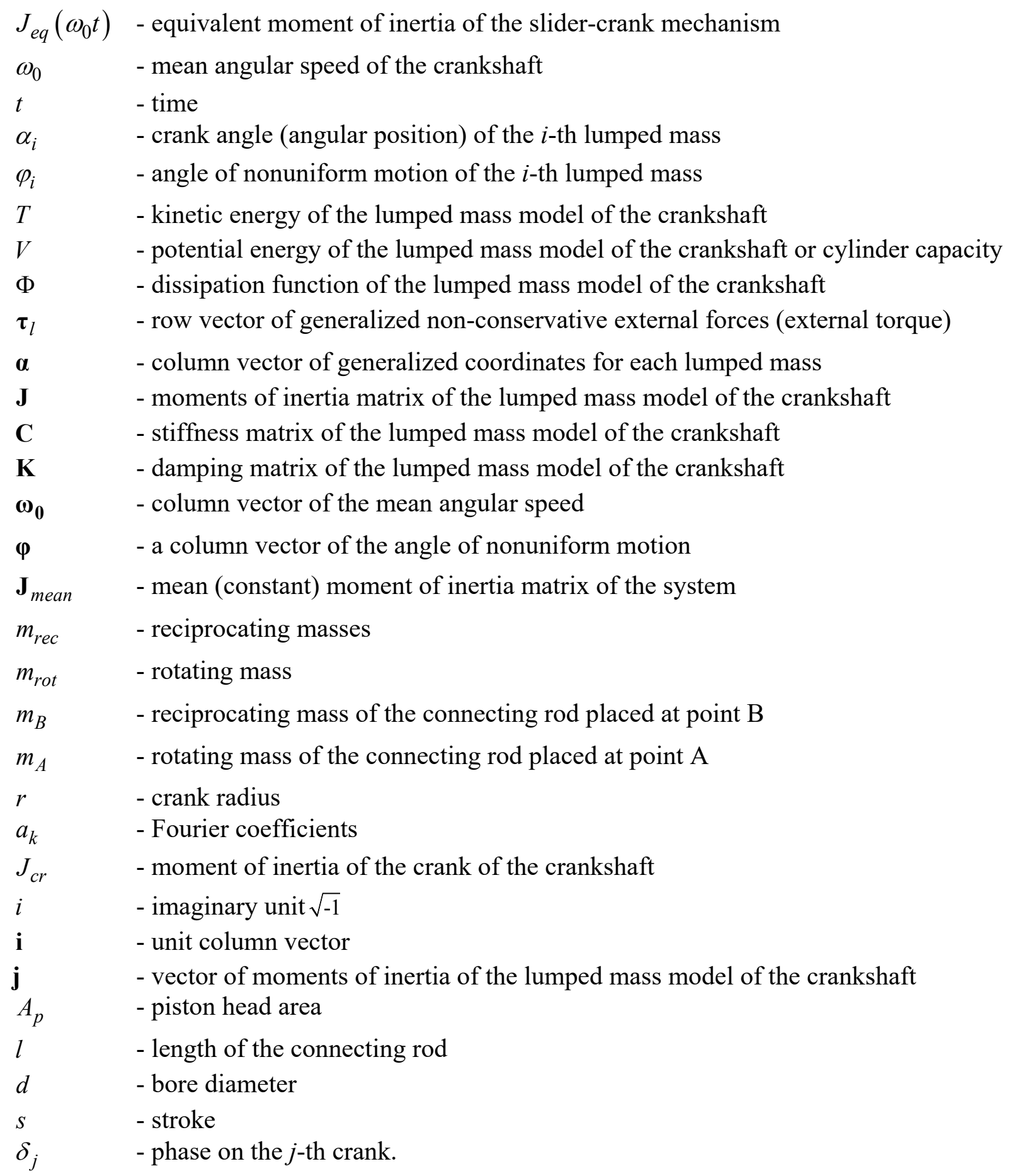


Determination of Differential Equations of Motion and Parameters of an Elastic

Internal Combustion Engine Crankshaft
A. Milašinović, Z. Milovanović, D. Knežević, I. Mujanić

\section{REFERENCES}

[1] Yamaguchi H.: Structural Dynamics, Asian Institute of Technology, Bankok \& Saitama University Urawa Japan, 1992.

[2] Beards C. E.: Structural Vibration: Analysis and Damping, John Wiley \&Sons, London, 1996.

[3] Mobley R. K.: Vibration fundamentals, Butterworth-Heinemann, Boston, 1999.

[4] Genta G., Vibrations of Structures and Machine, Springer-Verlag, New York, 1993. DOI: 10.1007/978-14757-1111-0

[5] Hafner K. E., Maass H., Torsionsschwingungen in der Verbrennungskraftmaschine, Springer-Verlag, Wien, 1985. DOI: 10.1007/978-3-7091-7002-1

[6] Haug, E.J., Computer-Aided Kinematics and Dynamics of Mechanical Systems, Volume I: Basic Methods, Allyn and Bacon, Needham Heights, Massachusetts, 1989.

[7] Filipović I., Kinematics and Dynamics of the IC Engine, Faculty of Mechanical Engineering Sarajevo, 1998.

[8] Shabana A. A., Dynamics of Multibody Systems, Cambridge University Press, Cambridge, 2005. DOI: $10.1017 / \mathrm{CBO} 9780511610523$

[9] Taraza D., Estimation of the Mean Indicated Pressure from Measurments of the Crankshafts Angular Speed Variation,. SAE Paper No. 932413, 1993.

[10] Taraza, D., Possibilities to Reconstruct Indicator Diagrams by Analysis of the Angular Motion of the Crankshaft, SAE Paper No. 932414, 1993.

[11] Schagerberg S., McKelvey T., Instantaneous Crankshaft Torque Measurements-Modeling and Validation, SAE Paper No 2003-01-0713, 2003.

[12] Chen S. K., Engine Diagnostics By Dynamic Shaft Measurement: a Progress Report, SAE Paper No. 932412, 1993.

[13] Zienkiewicz O.C, Taylor R.L. The Finite Element Method (5th edn), Vol. 1 - The Basis. ButterworthHeinemann: Oxford, 2000.

[14] Milašinović A., Filipović I.: Parametrically exicited torsional vibrations of crankshaft of internal combustion engine, Science and Motor Vehicle, International Automotive Conference YU-03101, Beograd 26.-28.05.2003.

[15] Milašinović A., Filipović I., Hribernik A.: Contribution to the definition of the torsional stiffness of the crankshaft of a diesel engine used in heavy-duty vehicles, Proceedings of the Institution of Mechanical Engineers, Part D: Journal of Automobile Engineering, Vol. 223, 921-930, London, 2009.

[16] Milašinović A. Mathematical modeling and experimental investigation of nonlinear torsional vibration of crankshaft of IC engine. PhD Thesis, University of Banja Luka, Bosnia and Herzegovina, April 2007.

[17] Brusa E., Delprete C., Genta G. Torsional vibration of crankshafts - effects of nonconstant moments of inertia, Journal of sound and vibration, 205(2), 1997, pp. 135-150. DOI: 10.1006/jsvi.1997.0964

[18] Milašinović A., Filipović I., Milovanović Z., Knežević D.: Determination of the Engine Torque of a Four Cylinder Four Stroke Diesel Engine on the Basis of Harmonic Analysis of the Crankshaft Angular Velocity, Transactions of Famena, University of Zagreb, Volume 35, No.4,55-63, Zagreb, 2011.

Submitted: $\quad 17.02 .2015$

Accepted: $\quad 10.5 .2016$
Prof. dr Aleksandar Milašinović Prof. dr Zdravko Milovanović Prof. dr Darko Knežević MSc Indir Mujanić Faculty of Mechanical Engineering University of BanjaLuka Stepe Stepanovića 71 78000 Banja Luka, Bosnia\&Herzegovina 